\title{
Culturing, In Vitro Microbial
}

National Cancer Institute

\section{Source}

National Cancer Institute. Culturing, In Vitro Microbial. NCI Thesaurus. Code C19347.

The growing of microbes in test tube or petri dish type environments 